\title{
Zählkontrolle im OP: Jeder Tupfer zählt!
}

\author{
Regina U. Dries, Synke Petschinka, Oliver Huwert, Norbert P. Südkamp
}

\section{Zusammenfassung}

Jeder Tupfer zählt! Dieser Appell vom Aktionsbündnis für Patientensicherheit e.V. ist bewusst plakativ gewählt, um auf die Standardisierung und Vereinheitlichung von Zählkontrollen im OP hinzuweisen. Das versehentliche Verbleiben eines Fremdkörpers nach operativen Eingriffen ist zwar eine seltene, doch allgemein bekannte, öffentliches Interesse erweckende Komplikation. Immer wieder wird auch in seriösen Tageszeitungen darüber berichtet. Diese Fehler entstehen häufig durch die verminderte Aufmerksamkeit und die ebenso gesenkte Detailwahrnehmung bei häufig getätigten Routinearbeiten, weiterhin stellt das Bestreben, möglichst schnell zum nächsten OP-Punkt übergehen zu können, eine Einflussgröße dar. Jede Einrichtung sollte deshalb schriftlich festlegen, wer für die Zählung verantwortlich ist, wer sie durchführt, was gezählt werden soll, wann, wer, was und wie die Ergebnisse dokumentiert werden und welche Konsequenzen aus den Ergebnissen abzuleiten sind. Besondere Situationen, wie z.B. vital bedrohliche Notfälle, die eine Abweichung von definierten Abläufen erfordern, sollten definiert und ebenfalls schriftlich festgelegt sein. Die Prävention und speziell das Thema der unbeabsichtigt im OP-Gebiet belassenen Fremdkörper sollten verbindlich in die Aus-, Weiter- und Fortbildung aller Gesundheitsberufe aufgenommen wer- den. Versehentlich belassene Fremdkörper werden auch in Zukunft ein besonderes Problem der Qualitätssicherung in der Chirurgie darstellen. Obwohl patienten- und operationsspezifische Risikofaktoren für das Belassen eines Fremdkörpers existieren, geht das Hauptrisiko immer noch von dem an der Operation beteiligten Personal aus. Hier gilt es, weiter Verbesserungen und Kontrollmechanismen zu entwickeln. Für eine erfolgreiche Zählkontrolle sind in der Durchführung wichtige Grundsätze zu berücksichtigen; Sicherheit braucht Zeit und Ruhe, Zählkontrolle ist immer Teamarbeit, geklärte Verantwortlichkeiten und Stopp bei Unklarheiten und eine sowohl unterstützende als auch schützende Dokumentation.

\section{Counting Controls in OP: Every Swab Counts}

Every swab counts! This appeal from the Action Committee for Patient Safety (Aktionsbündnis für Patientensicherheit e.V.) is deliberately poster-like to draw attention to the need for standardisation and uniformity in counting controls in the OP. Although the accidental forgetting of a foreign body after a surgical operation is a rare complication it does attract the general public's interest. Such reports occur frequently even in the more serious daily newspapers. Such errors often occur as a result of reduced concentration and equally of a lowered awareness of details in fre- quently performed routine work; furthermore, the urge to move on to the next operation may also be a relevant factor. Every institution should thus publish in written form just who is responsible for the counting control, who does it, what should be counted, how the results should be documented, and what consequences should be drawn from these results. Special situations such as, e.g., life-threatening emergencies that require a deviation from defined procedures, need to be defined and documented in written form. The prevention and, especially, the subject of inadvertently forgotten foreign bodies in the surgical site should without fail have a firm place in the primary education and continuing medical training of all medical and health-care personnel. Inadvertently forgotten foreign bodies will also in future continue to be a particular problem for quality assurance in surgery. Although patientand operation-specific risk factors for forgetting an object exist, the main risk resides in the personnel involved in the operation. Just for this reason further improvements and control mechanisms need to be developed. For a successful counting control, the following important basic principles must be observed: safety needs time and calmness, a counting control is always a team task, clearly defined responsibilities, an immediate stop in case of uncertainties, and a supporting as well as protecting documentation.
OP-JOURNAL 2010; 26: 214-218

(c) Georg Thieme Verlag KG Stuttgart · New York DOI http://dx.doi.org/10.1055/s-0030-1250557

\section{Einleitung}

Das versehentliche Verbleiben eines Fremdkörpers nach operativen Eingriffen ist zwar eine seltene, trotzdem vorkommende und öffentliches Interesse erweckende Komplikation. Immer wieder wird auch in seriösen Tageszeitungen darüber berichtet.
Beliebte Schlagzeilen sind:

- „Kompresse im Körper vergessen; Leben nach einem OP-Fehler" (RP online, 25.7.2007)

- „Patient verwechselt, Klemme vergessen“ (Berliner Zeitung, 5. 5.2006)

- „Ärztepfusch, wenn ein OP-Tuch zum Tumor wird“" (Stern, 4.11.2009) 


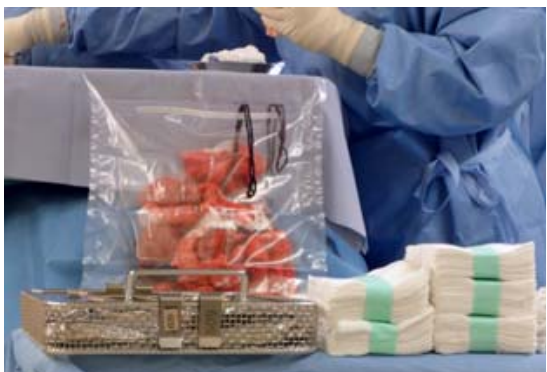

Abb. 1 Zählkontrollen tragen dazu bei, potenzielle Risiken für Patienten zu minimieren und insbesondere dafür Sorge zu tragen, dass die Patienten die OP-Abteilung nicht mit zurückgelassenen Materialien verlassen.

\section{- „3000-mal im Jahr wird OP-Werkzeug vergessen“"}

- „Ich habe es inzwischen raus, wie ich während einer OP für ein paar Minuten die Augen schließen und dösen kann, ohne dass es auffällt" (Stern, 2.9.2010)

Das Übersehen eines verbleibenden Fremdkörpers kann z.B. durch die gesenkte Aufmerksamkeit und/oder die gesenkte Detailwahrnehmung bei Routinearbeiten oder auch durch das Bestreben, möglichst schnell zum nächsten $\mathrm{OP}$ Punkt überzugehen, begünstigt werden.

Ca. 2000-mal pro Jahr geschieht es allein in Deutschland, dass Chirurgen oder OPPersonal im Körper eines Patienten einen Gegenstand vergessen und die Wunde darüber verschließen. Angesichts der hohen Operationszahlen ist das zwar immer noch äußerst selten, doch für die betroffenen Patienten steht die Gesundheit, oft sogar das Leben auf dem Spiel.

\section{Durchführung der Zähllkontrolle}

Bisher hat jedes Krankenhaus ein eigenes Vorgehen für das durchzuführende Zählprotokoll, hausintern wird definiert, welche Ver- und Gebrauchsgüter in der Zählkontrolle erfasst werden. Nicht alle Häuser erfüllen die geforderten oder empfohlenen Sicherheitsstandards.

Eine bundeseinheitliche Regelung zur Standardisierung und Dokumentation der Zählkontrolle im OP-Saal würde die Fehlerquote mit großer Wahrscheinlichkeit deutlich verringern und das Operationsteam rechtlich absichern.

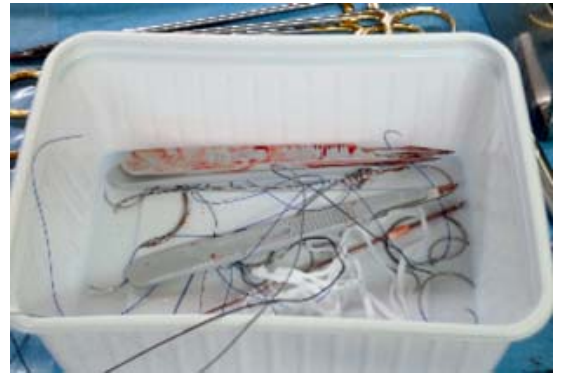

Abb. 2 Sicherheit am OP-Tisch. Für Nadeln und Spitzabfälle immer einen separaten Abwurfbehälter auf dem Tisch bereitstellen und einhalten. Ein sorgfältiges, einzelnes Durchzählen aller Verbandstoffe nach dem Entnehmen aus der Verpackung ist dringend anzuraten. Denn fehlerhaft bestückte Verpackungen sind nicht auszuschließen, hieraus können wiederum fatale Fehler resultieren.

\section{Juristische Vorgaben}

Jeder Patient schließt bei Eintritt in die Klinik einen Behandlungsvertrag mit der Klinik ab, d.h. bei der stationären Aufnahme wird zwischen Patient und behandelnden Personen ein Vertrag geschlossen; für diesen Behandlungsvertrag gelten die allgemeinen Geschäftsbedingungen. Aus diesem Vertrag ergibt sich die Verpflichtung auf eine korrekte und unversehrte Behandlung für den Patienten. Fehler müssen vermeidbar sein oder als vermeidbar gelten.

Aus juristischer Sicht haben Schutz und Sicherheit des Patienten oberste Priorität. Es müssen daher alle möglichen zumutbaren Sicherheitsvorkehrungen getroffen werden. Dazu gehören schriftliche Anweisungen für das instrumentierende Pflegepersonal bez. Zählkontrolle, Dokumentation, Schlussrevision und Beschreibung im OP-Bericht. Das Delegieren der Zählkontrolle an das Pflegepersonal ist zulässig, es führt jedoch zur Eigenverantwortung. Das bedeutet, dass ein Organisationsverschulden aufgrund des Vertrauensgrundsatzes ausscheidet. Trotz sorgfältigem Vorgehen stellt ein versehentlich belassener Fremdkörper einen vermeidbaren Fehler dar. Im Ereignisfall führt dies i.d. R. zur Haftung.

Als einzige Ausnahme wird der Umstand einer akuten lebensbedrohlichen Unübersichtlichkeit des Operationsfelds akzeptiert, für die jedoch die Beweislast gilt. Bei Außerachtlassen gebotener Maßnahmen wird der versehentlich belassene Fremdkörper aus juristischer Sicht als grober Behandlungsfehler gesehen.
Im Operationssal gibt es keine Unterscheidung für die verwendeten Materialien, somit ist eine belassene Schere im Körper des Patienten nicht schwerwiegender zu bewerten als eine vergessene Nadel. Vergessen ist vergessen.

Allerdings gilt auch: Solange nichts passiert, passiert nichts!

Eine Grundlage für die Ausbildung der Pflegenden im Operationsdienst bildet das Krankenpflegegesetz in der Fassung vom 4.6.1985. Dort werden u.a. in $\S 4$ „die sach- und fachkundige, umfassende Pflege des Patienten“ und „gewissenhafte Vorbereitung, Assistenz und Nachbereitung bei Maßnahmen der Diagnostik und der Therapie“ als Ausbildungsziele genannt.

Für die Arbeit im Operationsdienst bedeutet dies u.a.:

Fach- und sachkundiges, situationsgerechtes Instrumentieren, welches im Wesentlichen die Unterstützung der ärztlich-operativen Tätigkeit beinhaltet. Ein besonderer Aspekt dabei ist die hohe Verantwortung für die Sicherheit des Patienten in Bezug auf die Zählkontrolle benutzter Tupfer, Nadeln und anderer Materialien.

Jede Pflegeperson ist für die Sicherung der Qualität ihrer Tätigkeiten selbst verantwortlich!

Die Zählkontrolle ist eine der verantwortungsvollsten Aufgaben des OP-Personals!

Die Durchführungsverantwortlichkeit hierfür obliegt dem instrumentierenden Personal. Meist zählen Springer und Instrumentierender gemeinsam vor und nach dem Eingriff. Sie sind verpflichtet, den Operateur, der die Wunde nicht vor der Kontrolle der eingesetzten Produkte verschließen darf, sofort auf fehlende Instrumente oder Kompressen, Bauchtücher oder Tupfer etc. hinzuweisen. Da der Operateur im Verlauf der Operation meist keine Übersicht darüber hat, wie viele Verbandstoffe im Laufe einer OP zum Einsatz kamen, muss er sich auf das Pflegepersonal verlassen können, denn letztendlich trägt der Arzt die Endverantwortung für den durchgeführten Eingriff.

In manchen OP-Abteilungen ist es aus wirtschaftlichen Gründen zur Gewohnheit geworden, saubere und nicht ver- 


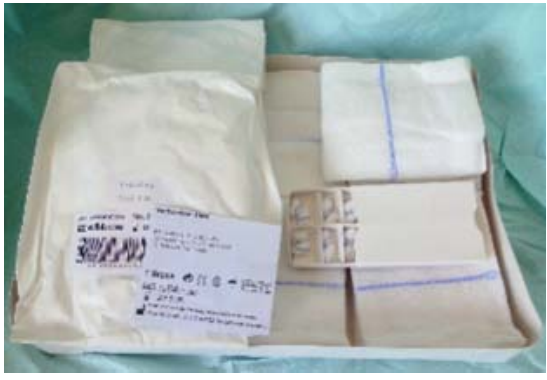

Abb. 3 Auch bei einem vorgefertigten Set gilt das Prinzip der Zählkontrolle, die ebenfalls über das Vier-Augen-Prinzip durchgeführt werden sollte.

wendete Bauchtücher und Kompressen als Putztücher zu benutzen. Hier besteht die zusätzliche Gefahr, dass solche als Reinigungstücher verwendete Bauchtücher in die abschließende Zählkontrolle geraten.

Solche Maßnahmen sind innerbetrieblich dringend zu überdenken!

Im Rahmen einer Umfrage bei OP-Pflegepersonal gaben 64\% der Befragten an, schon einmal an einer OP beteiligt gewesen zu sein, bei der durch die korrekt durchgeführte Zählkontrolle ein unbeabsichtigtes Zurücklassen eines Fremdkörpers verhindert wurde.

Darüber hinaus waren Stärken und potenzielle Schwächen zum Themenkomplex der Zählkontrolle erkennbar.

Positive Auswirkungen haben festgelegte Standards und Vorgehensweisen, gelebte Teamarbeit und die verantwortlich geleitete Einarbeitung von Mitarbeitern.

$\mathrm{Zu}$ den Schwächen gehören fehlende Routine, Zeitdruck, fehlende hausinterne Vorgaben, gesetzliche Vorgaben, die eine Zählkontrolle regeln.

Daher sollte das Zählen als Strategie zur Prävention unbeabsichtigt belassener Fremdkörper als gemeinschaftliche Aufgabe des OP-Teams aufgefasst und durchgeführt werden.

Die Arbeitsgruppe „Aktionsbündnis Patientensicherheit" empfiehlt in einer bundesweiten Kampagne die Durchführung einer standardisierten Zählkontrolle mit entsprechender Dokumentation bei Einhaltung eines gleichbleibenden Ablaufs und eindeutig verteilte Zuständigkeiten und Verantwortlichkeiten. Nachfolgend Auszüge aus den Empfehlungen.

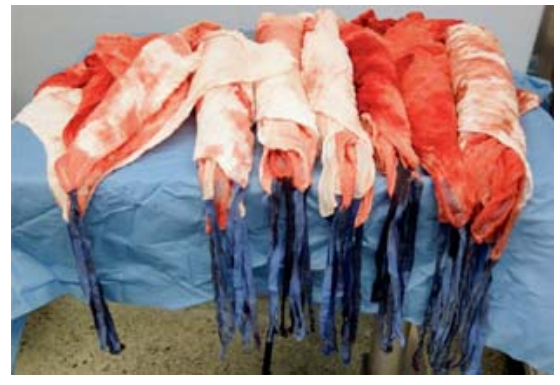

Abb. 4 Verwendete gezählte Bauchtücher, übersichtlich im 5er-Bündel gerollt. Zählung erfolgt durch das Vier-Augen-Prinzip.

\section{Zielsetzung}

Für alle Operationen sollen abteilungsspezifische Maßnahmen zur Prävention von unbeabsichtigt belassenen Fremdkörpern im OP-Gebiet definiert und allen beteiligten Mitarbeitern bekannt sein.

Jede Einrichtung sollte deshalb schriftlich festlegen

- wer für die Zählung verantwortlich ist,

- wer sie durchführt,

- was gezählt werden soll,

- wann, wer, was und wie die Ergebnisse dokumentiert werden,

- welche Konsequenzen aus den Ergebnissen abzuleiten sind.

\section{Klärung der Verantwortlichkeiten}

- Der Operateur trägt die Letztverantwortung für die Durchführung und für das Ergebnis des Zählens.

- Instrumentier- und Springerdienst tragen die Durchführungsverantwortung.

- Leitende Ärzte tragen die Verantwortung für die Anordnung der Zählkontrollen bzw. für die Maßnahmen zur Prävention.

- Die Krankenhausleitung ist verantwortlich für die Sicherstellung der erforderlichen materiellen, personellen, finanziellen und zeitlichen Ressourcen.

In besonderen Situationen, wie z.B. bei der Behandlung von Schwerstverletzten (Polytrauma) oder bei der Behandlung rupturierter Bauchaortenaneurysmen, kann es notwendig sein, von definierten Abläufen abzuweichen bzw. diese den veränderten Gegebenheiten anzupassen.

Z.B. kann es in vital bedrohlichen Notfällen erforderlich werden, übliche Zählkontrollen auszusetzen. Auch Teamwechsel während einer OP führen zu
Änderungen in den Operationsabläufen und stellen ein zusätzliches Risiko dar. Denn die meisten Fehler passieren durch Unaufmerksamkeit oder Ablenkungen. Deshalb sollte definiert werden, wann und unter welchen Bedingungen ein Teamwechsel durchgeführt wird.

Auftretende Sondersituationen sollten ebenfalls schriftlich festgelegt sein.

\section{Besonders wichtig:}

- klare unmissverständliche Vorgaben, wie im Fall diskrepanter Zählergebnisse vorzugehen ist,

- welche Maßnahmen im Schadensfall einzuleiten sind,

- wie Patienten, die einen unbeabsichtigt belassenen Fremdkörper bei sich vermuten, zu behandeln sind.

Das Aktionsbündnis regt dringend an, Prävention und speziell das Thema der unbeabsichtigt im OP-Gebiet belassenen Fremdkörper verbindlich in die Aus-, Weiter- und Fortbildungen aller Gesundheitsberufe aufzunehmen.

Zählkontrollen sind präoperativ, intraoperativ und postoperativ durchzuführen.

Jeder Zählschritt sollte von 4 Augen (instrumentierende Pflegekraft + Springerdienst) kontrolliert werden. Zu zählen sind je nach Fachdisziplin z.B.:

- Instrumentensiebe

- Zusatzinstrumente

- Nadeln und Nadelfadenkombinationen (Nadeln immer in ein geeignetes Behältnis entsorgen, sie sollten nie allein auf dem Tisch herumliegen)

- röntgenpositive Verbrauchsmaterialien wie Bauchtücher, Tupfer, Kompressen, Präpariertupfer

- nicht röntgenpositive Materialien wie z. B. Zügel, Spritzen

- Implantate

Die Vorbereitung der Instrumentiertische erfolgt nach abteilungsspezifischen Vorgaben. Begründete Abweichungen bei der Anzahl der Instrumente und der Verbandstoffe werden unverzüglich dem Springer mitgeteilt und durch diesen im Zählprotokoll dokumentiert.

Diese Ergebnisse werden nach abteilungsspezifischer Vorgabe dokumentiert. Das Ziel muss sein, dass alle zum Einsatz kommenden Materialien bekannt, gezählt und dokumentiert sind. 


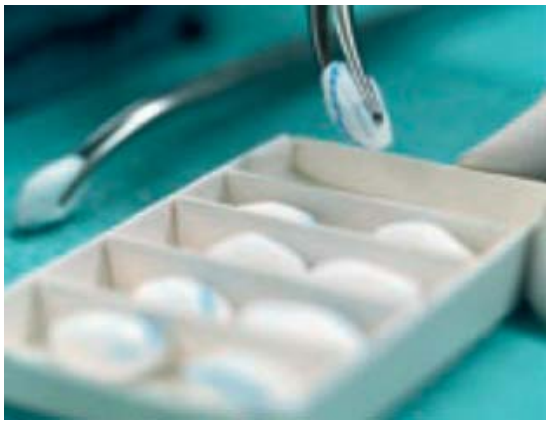

Abb. 5 Die Präpariertupfer (z. B. von der Fa. Hartmann) sind in einer Schiebeschachtel mit Rasterung. So lassen sich die kleinen Präpariertupfer besser handhaben, v.a. aber hat man die genaue Anzahl der Tupfer stets vor Augen. Die Tupfer sind röntgenarmiert.
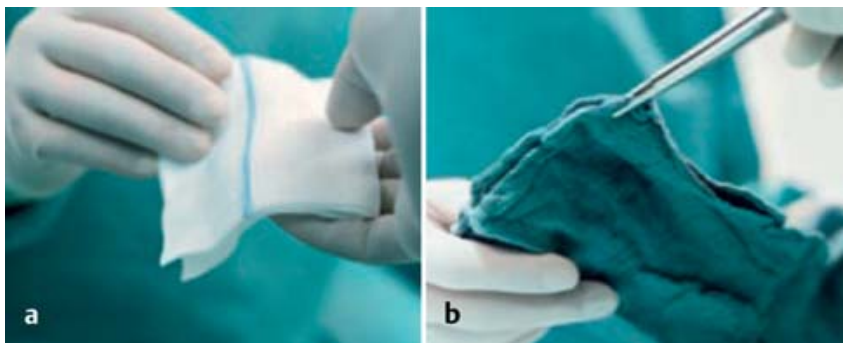

Abb. $7 \mathrm{a}$ und $\mathrm{b}$ Ebenso sind (a) Kompressen und (b) Bauchtücher mit einem Röntgenkontrastmedium ausgerüstet, um den Verbleib in situ durch eine Röntgenkontrolle ausschließen zu können.

Für zusätzliche Sicherheit, sowohl für den Chirurgen als auch den Patienten, ist die ausschließliche Verwendung von Verbandstoffen mit einem Röntgenkontrastmedium erforderlich. So besteht die Möglichkeit, bei einem abweichenden Zählergebnis durch eine sofortige Röntgenkontrolle den Verbleib von Verbandstoffen auszuschließen bzw. kann gegebenenfalls die Lage des zurückgelassenen Verbandstücks exakt bestimmt werden.

Intraoperativ sind alle zusätzlich angereichten Materialien fortlaufend unter dem Vier-Augen-Prinzip zu zählen und zu dokumentieren.

Der Instrumentierdienst überprüft intraoperativ stets alle Materialien, die vom Chirurgen zurückgegeben werden, auf Vollständigkeit.

Jedes Teammitglied muss Zweifel an der Vollständigkeit sofort laut und deutlich aussprechen!

Grundsätzlich erfolgt die offizielle Zählkontrolle vor dem Verschluss von Körperhöhlen und Organen, vor dem Wundverschluss, vor bzw. während der Hautnaht. Selbstverständlich sollte bei jedem Teamwechsel der Pflegekraft eine offizielle Zählkontrolle durchgeführt werden.
Die Ergebnisse werden vom Springerdienst dokumentiert, danach wird der Operateur am Tisch informiert, dieser bestätigt die Ansage.

Die instrumentierende Pflegekraft und der Springerdienst überprüfen bei der postoperativen Zählkontrolle letztmalig die Vollständigkeit der Materialien, die endgültige Entsorgung erfolgt erst nach Abschluss und Dokumentation der postoperativen Zählkontrolle. Die Zählergebnisse werden zeitnah von der instrumentierenden Pflegekraft und dem Springer auf dem Zählprotokoll abgezeichnet. Ebenso sollten Besonderheiten und zusätzliche Zählkontrollen dokumentiert und abgezeichnet sein.

Sind beabsichtigt belassene Fremdkörper im Wundgebiet (z.B. Tamponaden durch Bauchtücher), muss die Anzahl und die Lokalisation durch den Springer und den Operateur dokumentiert werden.

Sollten Diskrepanzen bei der Zählkontrolle entstehen, müssen klare Richtlinien für die weitere Vorgehensweise bestehen:

- die Prozedur wird gestoppt

- der Operateur entscheidet über das weitere Vorgehen

- systematische Revision des Wundgebiets
- z.B. intraoperatives Röntgen

- Dokumentation

\section{Risiken}

Die großen Fachbereiche Orthopädie und Unfallchirurgie, Viszeralchirurgie, Herzchirurgie, Urologie sowie Gynäkologie und Geburtshilfe gelten als besonders risikobehaftet.

Erhöhte Risikofaktoren für das Zurückbleiben von Fremdkörpern bestehen bei folgenden Situationen (nach [2]):

- Notfalleingriffe

- unerwartete Änderungen im OP-Ablauf

- Einsatz mehrerer chirurgischer Teams

- Wechsel des Pflegepersonals

- hoher Body-Mass-Index des Patienten

- hoher Blutverlust während der OP

Ein weiterer Risikofaktor dürfte allerdings auch der immer größer werdende Zeitdruck und die Arbeitsverdichtung im OP sein. Hinzu kommen unzureichende Standards, Unwissenheit und Fehlinformation des Pflegepersonals.

Hausinterne Dokumente oder Verfahrensanweisungen, die das Verfahren der Zählkontrolle beschreiben, sollten für die Mitarbeiter jederzeit zugänglich sein.

Versehentlich belassene Fremdkörper nach [6]:

- Bauchtücher

- Tupfer

- Drainagen/-teile

- Katheter/-teile

- Nadeln

- Unterlegscheiben

- OP-Klemmen

- Tamponaden

- Markierungsdrähte

- Endoskopieteile

- Zangenteile

- Metallklammern

- Instrumente

- Schere

- Bohrer/-teile

- Metallspatel

- Retraktoren

- Sauger

\section{Vorschläge zur Prävention}

Die Projektgruppe des „Aktionsbündnisses Patientensicherheit" schlägt zur Prävention unbeabsichtigt belassener Fremdkörper im OP-Gebiet folgende Punkte zur Umsetzung in den Kliniken vor: 
Interdisziplinäre und berufsgruppenübergreifende Erarbeitung und schriftliche Festlegung

- der Verantwortlichkeiten und der Aufgaben der beteiligten Personen,

- der Zählmethode,

- zur Art und Beschaffenheit der zu zählenden Materialien (z.B. röntgenpositiv),

- des Umfangs, der Häufigkeit und der Zeitpunkte der Zählkontrollen,

- der Vorgehensweise bei einem Teamwechsel,

- der Vorgehensweise bei Diskrepanzen bei den Zählergebnissen,

- der Vorgehensweise bei beabsichtigt belassenen Materialien,

- der Vorgehensweise bei vital bedrohlichen Notfällen (Aussetzen von Zählkontrollen),

- des Umgangs mit Materialien, welche während einer OP zur Entsorgung anfallen,

- der Dokumentation.

Darüber hinaus empfiehlt die Arbeitsgruppe die Erarbeitung von einheitlichen schriftlichen Festlegungen zum Umgang mit

- Beinaheschäden,

- dem eingetretenen Ereignis eines unbeabsichtigt belassenen Fremdkörpers,

- Patienten, die vermuten, dass bei ihnen unbeabsichtigt ein Fremdkörper belassen wurde,

- beabsichtigt belassenen Fremdkörpern.

\section{Ausblick}

Versehentlich belassene Fremdkörper werden auch in Zukunft ein besonderes Problem der Qualitätssicherung in der Chirurgie darstellen. Obwohl patientenund operationsspezifische Risikofaktoren für das Belassen eines Fremdkörpers existieren, geht das Hauptrisiko immer noch von dem beteiligten Personal an der Operation aus. Hier gilt es, weitere Verbesserungen und Kontrollmechanismen zu entwickeln [3].

Weiters gilt es, die Thematik in den Ausbildungsberufen zu behandeln und durch gezielte theoretische und praktische Schulungen Prävention zu betreiben. Im straff organisierten Operationsbetrieb ist $\mathrm{zu}$ berücksichtigen, dass korrekt durchgeführte Zählkontrollen immer Zeit brauchen. Die gewissenhafte Dokumentation schützt vor Unklarheiten.

Nicht zu vergessen: Zählkontrolle ist Teamarbeit. Alle Beteiligten im Operationssaal sind hierzu aufgerufen, denn „Jeder Tupfer zählt".

\section{Literatur}

${ }^{1}$ Aktionsbündnis für Patientensicherheit. Jeder Tupfer zählt! - Handlungsempfehlungen zur Vermeidung unbeabsichtigt belassener Fremdkörper im OP-Gebiet. Im Internet: www.aktionsbuendnis-patientensicherheit. de, info@aktionsbuendnis-patientensicherheit.de; Dezember 2009

2 Schindle B, Mutz A, Mousissiau M et al. Arbeitsanweisung Zählkontrolle im OP. Freiburg: Universitätsklinikum Freiburg; 2005

${ }^{3}$ Hartmann. Das Telatrast Modul-System: für ein Maximum an Sicherheit. OP-News Zeitschrift für OP-Personal und Ärzte 2008; 5: 4-5

${ }^{4}$ Gawande A, StuddertD, Orav Jet al. Risk factors for retained instruments and sponges after surgery. N Engl J Med 2003; 348: 229-235

${ }^{5}$ Lang JM, Uhl E. Der versehentlich belassene Fremdkörper: ein unbeabsichtigtes Implantat. Journal für Neurologie, Neurochirurgie und Psychiatrie 2009; 10: 14-17

${ }^{6}$ Reim W. Qualitätssicherung im OP. Im Internet: http://www.pflege-im-op.de/Praxisberichte/sonstige/Qualitaetssicherung/qualitaetssicherung.html; 12.7.2008

7 Ulsenheimer K. Belassene Fremdkörper aus Sicht des Juristen. Chirurg 2007; 78: 28-34

8 Petry FM. Aus medizinischen Behandlungsfehlern für die zukünftige Patientensicherheit lernen? 38. Berliner Krankenhaus-Seminar; 07.11.2007

${ }^{9}$ Stern Magazin. Risiko Krankenhaus. Nr.36, 2.9. 2010
Regina U. Dries

OP-Gesamtleitung, Zentral OP

Chirurgische Klinik

Universitätsklinikum Freiburg

Hugstetter Str. 55

79106 Freiburg

regina.dries@uniklinik-freiburg.de

\section{Synke Petschinka}

OP-Pflege

OP-Abteilung

Klinikum Bielefeld

An der Rosenhöhe 27

33647 Bielefeld

Oliver Huwert

Assistenzarzt

Department Orthopädie

und Unfallchirurgie

Universitätsklinikum der

Albert-Ludwigs-Universität Freiburg

Hugstetter Straße 55

79106 Freiburg

Univ.-Prof. Dr. med. Dipl.-Ing.

Norbert P. Südkamp

Geschäftsführender Direktor

Department Orthopädie

und Traumatologie

Universitätsklinikum der

Albert-Ludwigs-Universität Freiburg

Hugstetter Straße 55

79106 Freiburg 